\title{
Numerical analysis of pressure fluctuation in a multiphase rotodynamic pump with air-water two-phase flow
}

\author{
Wenwu Zhang, Zhiyi Yu*, Yongjiang Li, Jianxin Yang, and Qing Ye \\ School of Mechanical Engineering, Beijing Institute of Technology, Beijing 100081, China
}

Received: 12 September 2018 / Accepted: 12 December 2018

\begin{abstract}
Pressure fluctuation in single-phase pumps has been studied widely, while less attention has been paid to research on multiphase pumps that are commonly used in the petroleum chemical industry. Therefore, this study investigates the pressure fluctuation for a multiphase rotodynamic pump handling air-water two-phase flow. Simulations based on the Euler two-fluid model were carried out using ANSYS CFX16.0 at different Inlet Gas Void Fractions $(I G V F \mathrm{~s})$ and various flow rate values. Under conditions of $I G V F=0 \%$ (pure water) and $I G V F=15 \%$, the accuracy of the numerical method was tested by comparing the experimental data. The results showed that the rotor-stator interaction was still the main generation driver of pressure fluctuation in gas-liquid two-phase pumps. However, the fluctuation near the impeller outlet ascribe to the rotor-stator interaction was weakened by the complex gas-liquid flow. For the different IGVF, the variation trend of fluctuation was similar along the streamwise direction. That is, the fluctuation in the impeller increased before decreasing, while in the guide vane it decreased gradually. Also, the fluctuation in the guide vane was generally greater than for the impeller and the maximum amplitude appeared in the vicinity of guide vane inlet.
\end{abstract}

\section{Nomenclature}

Amp Amplitude, $\mathrm{kPa}$

$C_{p} \quad$ Pressure coefficient, dimensionless

FFT Fast Fourier Transform

$H \quad$ Head, $\mathrm{m}$

$H_{\mathrm{d}} \quad$ Designed head, $\mathrm{m}$

$H_{\mathrm{g}} \quad$ Head of gas, $\mathrm{m}$

$H_{1} \quad$ Head of water, $m$

$H_{\mathrm{t}} \quad$ Test pump head

IGVF Inlet Gas Void Fraction, dimensionless

$n \quad$ Rotational speed, $\mathrm{r} / \mathrm{min}$

$p \quad$ Static pressure, $\mathrm{kPa}$

$p_{\mathrm{i}} \quad$ Static pressure at the pump inlet, $\mathrm{Pa}$

$p_{\mathrm{i}}(t) \quad$ Instantaneous static pressure, $\mathrm{Pa}$

$\bar{p}_{i} \quad$ Average static pressure, $\mathrm{Pa}$

$p_{\mathrm{o}} \quad$ Static pressure at the pump outlet, $\mathrm{Pa}$

$P \quad$ Shaft power, $\mathrm{kW}$

$P_{\mathrm{e}} \quad$ Effective power, $\mathrm{kW}$

$Q_{\mathrm{d}} \quad$ Designed flow rate, $\mathrm{m}^{3} / \mathrm{h}$

$v_{i} \quad$ Velocity at pump inlet, $\mathrm{m} / \mathrm{s}$

$v_{\mathrm{o}} \quad$ Velocity at pump outlet, $\mathrm{m} / \mathrm{s}$ $\begin{array}{ll}w_{l s} & \text { Water superficial velocity, } \mathrm{m} / \mathrm{s} \\ \Delta t & \text { Time step, } \mathrm{s}\end{array}$

\section{Greek symbols}

$\alpha_{\mathrm{g}} \quad$ Gas void fraction, dimensionless

$\beta \quad$ Mass fraction of gas, $\%$

$\eta \quad$ Efficiency, dimensionless

$\eta_{\mathrm{t}} \quad$ Test pump efficiency, dimensionless

$\rho \quad$ Density, $\mathrm{kg} / \mathrm{m}^{3}$

$\sigma \quad$ Standard deviation of pressure, $\mathrm{Pa}$

\footnotetext{
* Corresponding author: yuzhiyi@bit.edu.cn
}

$\begin{array}{ll}\text { Subscripts } \\ \text { d } & \text { Design } \\ \text { e } & \text { Effective } \\ \text { g } & \text { Gas } \\ \text { i } & \text { Inlet } \\ \text { l } & \text { Liquid } \\ \text { o } & \text { Outlet } \\ \text { t } & \text { Test }\end{array}$




\section{Introduction}

Pressure fluctuation in pumps not only increases vibration of the unit, but can also increase noise levels and cavitation, seriously affecting the safety and stability of the pump (Chen et al., 2016; Hayashi and Kaneko, 2014; Nishida et al., 2016) and is therefore an important aspect for study. For single-phase pumps, many factors cause pressure fluctuation, including cavitation, flow separation, vortex hand, and rotor-stator interaction but the rotor-stator interaction is viewed as the major cause (Miorini et al., 2012). However, for gas-liquid two-phase pumps, the flow is usually more disordered due to the phase interaction between the two phases effecting the fluctuation in such pumps (Liu et al., 2017a; Ma et al., 2018; Yu et al., 2014).

Many studies on the pressure fluctuation in single-phase pumps have been conducted or even carried out in-depth cavitation analysis in recent years, including for centrifugal pumps (González et al., 2006; Liu et al., 2017b; Majidi, 2005), axial flow pumps (Feng et al., 2016; Shuai et al., 2014; Xie et al., 2018) and mixed-flow pumps (Miyabe et al., 2006; Xu et al., 2017; Zhang et al., 2017b). González et al. (2006) investigated pressure fluctuation and radial forces for a centrifugal pump with an impeller to tongue gap variation and obtained the dynamic forces at the blade passing frequency. While Feng et al. (2016) found that the effect of clearance variation on the fluctuation in the impeller is larger than for the guide vane for an axial flow pump, which agreed with Zhang et al.'s (2017a) analysis for a mixed-flow pump. Additionally, by exploring the pressure fluctuation in a mixed-flow pump under cavitation condition, $\mathrm{Xu}$ et al. (2017) found a correlation between the dominant frequency and the variation of the cavitation regions. Thus, despite the type of single-phase pump, the rotor-stator interaction is always seen as the main generation driver of pressure fluctuation. In gas-liquid two-phase pumps, due to the phase interaction, flow separation and the mixing of the two phases, the flow may become disordered and the flow pattern variable with the operating conditions of pump. However, research on such pumps has been focused primarily on design optimization (Cao et al., 2005; Kim et al., 2015; Suh et al., 2017) and transport properties (Minemura and Murakami, 1980; Minemura and Uchiyama, 1993). In terms of the pressure fluctuation, although Zhang et al. (2017c) and Tan and Zhang (2018) made a preliminary analysis, the relationship between the fluctuation and $I G V F$ as well as the effect of flow rate was not discussed.

With the technological development of Computational Fluid Dynamics (CFD), CFD simulation has become a primary tool to investigate the internal flow in multiphase pumps (Caridad and Kenyery, 2004; Pineda et al., 2016). Also, the Euler two-fluid model has been applied more widely in the related simulation because of its higher calculation precision than other two-phase models such as mixture model and drift flow model, among others (Suh et al., 2018, Zhang et al., 2018a). In this model, each medium has its own governing equations, that is, each medium has its own velocity field but share the same pressure field and the phase interaction between the two phases is considered (Lane et al., 2005; Zhang et al., 2018b; Yan et al., 2017;
Yu et al., 2015). Based on the Euler two-fluid model, the biphasic characterization of a centrifugal pump was investigated by Caridad et al. (2008), and they showed that an increase in bubble diameter will be detrimental to the pump head. While based on the Euler two-fluid model and a developed mechanistic model, Zhu and Zhang (2016) predicted the in-situ GVF in an electrical submersible pump impeller and obtained that with the increase of bubble diameter and the decrease of gas density and rotation speed, the in-situ GVF increased.

On the whole, few studies on the pressure fluctuation in multiphase pumps have been carried out, thus the main cause of pressure fluctuation and its change rules in such pumps need further exploration. In this study, an experimental system of the multiphase pump handling air-water two-phase flow was described firstly; secondly, the reliability of the numerical methods was discussed in terms of turbulence models, structured mesh, steady and unsteady simulations; then the unsteady simulations based on the Euler two-fluid model were carried out using ANSYS_CFX 16.0 at $I G V F=15 \%$. In addition, because of the complexity of the operation conditions, the multiphase pumps may operate at different Inlet Gas Void Fractions $(I G V F \mathrm{~s})$ and various flow rates. Therefore, the influences of $I G V F$ and flow rate were investigated. The objective of this research is to obtain the mechanism of fluctuation and determine the change rules.

\section{Experimental system of the multiphase pump}

\subsection{Experimental design}

An experimental system diagram of the gas-liquid twophase pump is shown in Figure 1. To ensure the safety of experiment and the reusability of equipment, as well as to observe the bubble diameter and the internal flow in the pump more conveniently, air and water were therefore selected. Meanwhile, before entry into the multiphase pump, they were mixed evenly in a mixer to provide a bubbly flow in the inlet pipe. The central perforated tube is the most important component of the mixer. The gas breaks and mixes uniformly with the liquid in the central perforated tube and the bubble size is adjusted by the openings in it. The mixer, multiphase pump, and water tank are labelled 4, 5, and 1 respectively in Figure 1. A schematic diagram of the test system has been present as Figure 2 to provide a better understanding of the real diagram. In addition, to observe the internal flow of the multiphase pump, the casing of impeller and guide vane was made from Plexiglas and the maximum pressure available on it is $0.6 \mathrm{MPa}$, as shown in Figure 3.

The test pump head is obtained by equations (1)-(3), where $\beta$ is the mass fraction of gas; $p_{\mathrm{i}}$ and $p_{\mathrm{o}}$ are the pressure at inlet and outlet of the pump respectively measured by the pressure gauge with an accuracy of $\pm 0.25 \%$; $v_{\mathrm{i}}$ and $v_{\mathrm{o}}$ are the velocities at the pump inlet and outlet respectively. The velocities of air and water are calculated based on the flow rates, which are measured by the 
rotameter and the turbine flowmeter respectively, with an accuracy grade of $\pm 0.5 \%$ :

$$
\begin{gathered}
H_{\mathrm{t}}=(1-\beta) H_{\mathrm{l}}+\beta H_{\mathrm{g}}, \\
H_{\mathrm{l}}=\frac{p_{\mathrm{o}}-p_{\mathrm{i}}}{p_{\mathrm{l}} g}+\frac{\left(v_{0}^{2}-v_{i}^{2}\right)_{1}}{2 g}, \\
H_{\mathrm{g}}=\frac{p_{o}-p_{i}}{p_{\mathrm{g}} g}+\frac{\left(v_{0}^{2}-v_{i}^{2}\right)_{\mathrm{g}}}{2 g} .
\end{gathered}
$$

The test pump efficiency is computed by equations (4)-(5), where $Q$ denotes the total void flow rate of the two phases; $P$ is the shaft power of the pump and is measured by a torque power meter with an accuracy of $\pm 0.2 \%$. In addition, according to the calculation method used in the Zhang et al. (2015), the measurement error of pump head and efficiency can be determined to be about $0.8 \%$ :

$$
\begin{gathered}
\eta_{\mathrm{t}}=\frac{P_{\mathrm{e}}}{P}, \\
P_{\mathrm{e}}=\frac{\rho g Q H_{\mathrm{t}}}{1000},
\end{gathered}
$$

here, the density $\rho$ is the mixture density defined by:

$$
\rho=\rho_{\mathrm{g}} I G V F+\rho_{1}(1-I G V F) .
$$

\subsection{Parameter specifications}

The multiphase pump in this study includes four components, i.e. the inlet and outlet pipes, impeller, and guide vane. Its designed rotational speed $n$ is $2950 \mathrm{r} / \mathrm{min}$; designed flow rate $Q_{\mathrm{d}}$ is $50 \mathrm{~m}^{3} / \mathrm{h}$; and designed head $H_{\mathrm{d}}$ is $15 \mathrm{~m}$. The lengths of the inlet and outlet pipes are $300 \mathrm{~mm}$ and $400 \mathrm{~mm}$, respectively. The number of blades of the impeller and guide vane is four and eleven respectively and other design parameter specifications for the impeller and guide vane passages are shown in Figure 4.

\section{Numerical methods}

\subsection{Governing equations}

Two-fluid model was adopted in this study to predict the internal flow of the multiphase pump. Furthermore, the drag model considered to be the most important phase interaction model was modified using the secondary development technology of ANSYS_CFX16.0, which has been introduced in the published literature (Zhang et al., 2019). In addition, the basic governing equations for incompressible flow in the multiphase rotodynamic pump have been detailed in previous studies (Yan et al., 2017; Yu et al., 2015; Zhang et al., 2017c). Significantly, as a result of a lack of an in-depth understanding of the internal flow mechanisms in gas-liquid two-phase pumps, no one turbulence model has been applied widely and accurately to predict the complex flows in such pumps (Franke, 2009).

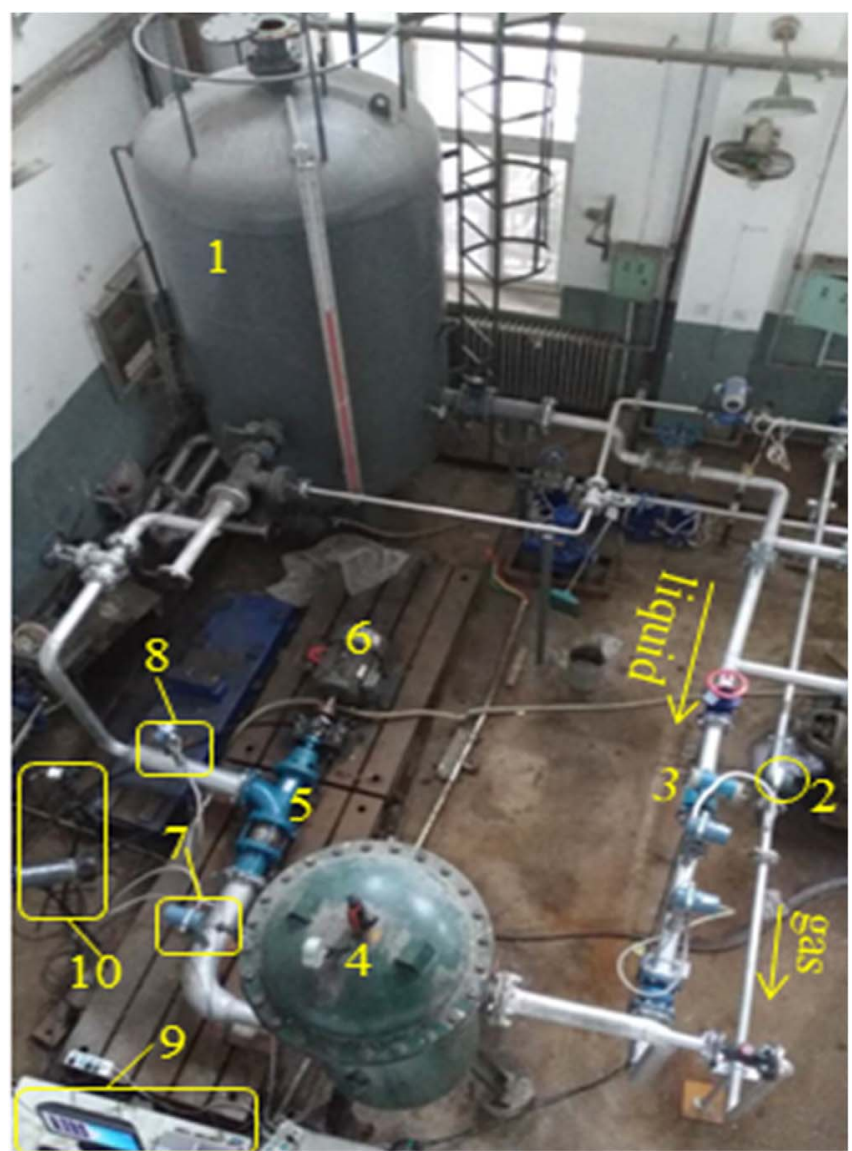

Fig. 1. Experimental system diagram of the multiphase pump. 1. Tank; 2. Rotameter; 3. Turbine flowmeter; 4. Mixer; 5. Multiphase rotodynamic pump; 6. Electric motor; 7, 8. Pressure gauges; 9. Collecting system; 10. High-speed camera and lighting.

Usually, two-equation turbulence models, such as

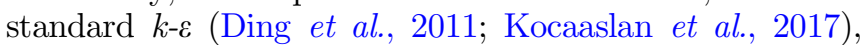
Renormalization Group (RNG) $k-\varepsilon$ (Qu et al., 2016; Tan et al., 2014), $k-\omega$ (Feng et al., 2010; Menter, 1994), and

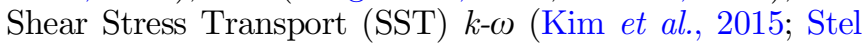
et al., 2015; Yan et al., 2017; Yu et al., 2015; Zhang et al., 2017c), have been used as they have a better compromise in terms of numerical effort and computational accuracy. Here, the SST $k-\omega$ model is a blending model between the $k-\omega$ model near the wall and the $k-\varepsilon$ model in the outer region. Therefore, the SST $k$ - $\omega$ model can account for the transport of the turbulent shear stress and give a highly accurate prediction of flow separation under adverse pressure gradients (Zhang, 2000).

The values of numerical efficiency and head at these four turbulence models along with the experimental results are listed in Table 1. It shows that the results for the standard $k-\varepsilon$ model are furthest from the experiment, which may ascribe to the inaccurate $\varepsilon$ equation, especially for the flow with large strain rate. In fact, this is also why Yakhot et al. (1992) developed the RNG $k-\varepsilon$ model. From Table 1, although the results for the RNG $k-\varepsilon$ and SST $k$ - $\omega$ models are close to the experimental results, the SST $k-\omega$ model 


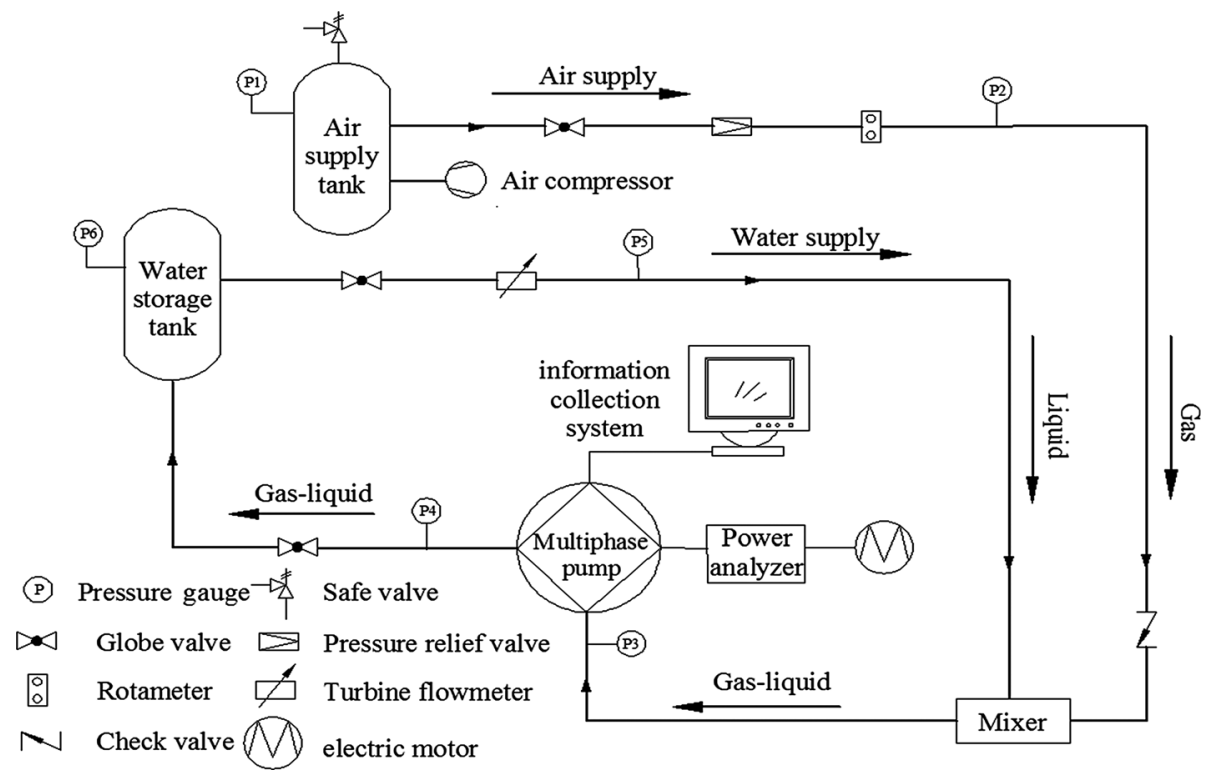

Fig. 2. Schematic diagram of the test system.

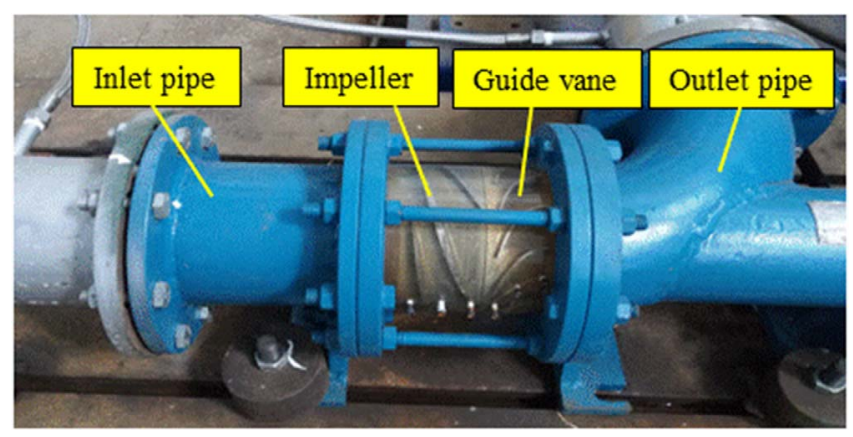

Fig. 3. Experimental pump model.

was chosen for the present study as a result of the complex gas-liquid flow and the existence of adverse pressure gradient in the multiphase pump.

\subsection{Structured mesh}

Figure 5 shows the three-dimensional numerical pump model with a full flow passage and the structured mesh adopted for each component. In order to ensure the mesh qualities of the impeller and guide vane, an Automatic Topology and Meshing (ATM) optimized topology was applied in ANSYS TurboGrid (SAS IP, Inc., 2013). This topology type creates high-quality meshes by adjusting the global size factor as well as the distribution parameters in the hub and shroud layers. The meshes for the impeller and guide vane, as well as the partial enlarged view, are shown in Figure 6.

The pump head and efficiency are compared with four mesh numbers under pure water conditions $(Q=$ $49.3 \mathrm{~m}^{3} / \mathrm{h}$ ) to efficiently utilize the computer resources and

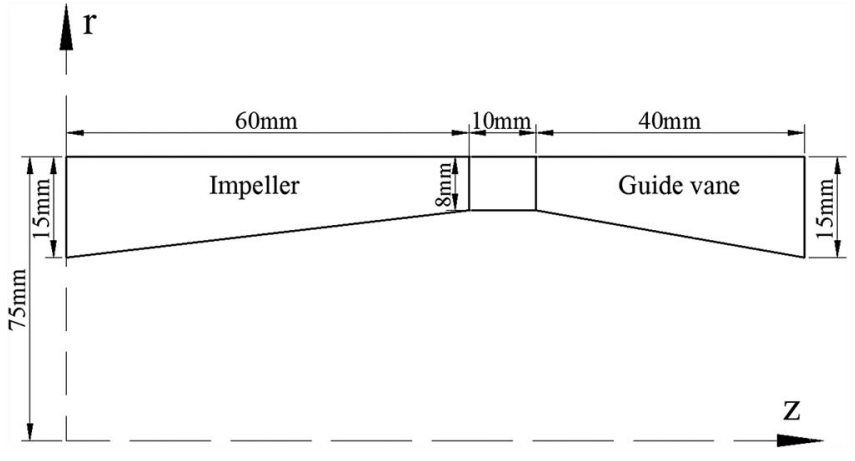

Fig. 4. Parameter specifications for the impeller and guide vane passages.

to improve the computational efficiency as shown in Figure 7. This shows that the differences in head and efficiency are smaller between 3.68 million and 4.82 million mesh numbers, thus the total mesh number of 3.68 million was adopted finally.

The detailed mesh information for the impeller and guide vane, such as elements, nodes, orthogonality, expansion factors, Aspect ratios, and $y+$ is extracted and listed in Table 2 . The orthogonality angle range is $0^{\circ}-90^{\circ}$ with larger values indicating better orthogonality. Expansion involves the ratio of the maximum to minimum distance between the control volume node and the control volume boundaries with an acceptable range of 1-20 (SAS IP, Inc, 2013). Aspect ratio relates to the ratio of the maximum to minimum integration point surface areas in all elements adjacent to a node with an acceptable range of 1-100 (SAS IP, Inc, 2013). The $y+$ value is a non-dimensional 
Table 1. Comparison of the efficiency and head between simulation and experiment $\left(Q=Q_{\mathrm{d}}\right)$.

\begin{tabular}{lccccr}
\hline Item & \multicolumn{4}{c}{ Simulation } & Experiment \\
\cline { 2 - 5 } & Standard $k-\varepsilon$ & RNG $k-\varepsilon$ & $k-\omega$ & SST $k-\omega$ & \\
\hline Efficiency $\eta(\%)$ & 60.78 & 58.07 & 60.03 & 58.04 & 57.50 \\
Head $H(\mathrm{~m})$ & 17.97 & 17.05 & 17.78 & 17.05 & 16.55 \\
\hline
\end{tabular}

parameter representing the distance from the wall to the first node away from the wall (SAS IP, Inc, 2013). Thus, according to the analysis shown in Table 2 , it can be concluded that the mesh qualities in this study can meet the requirements of unsteady simulation.

\subsection{Simulations and validations}

\subsubsection{Steady simulation}

Because of its computational efficiency and acceptable computational accuracy for steady simulation, the external characteristics of pumps, such as efficiency and head, are predicted by steady simulation (Kim et al., 2015; Tan et al., 2014; Zhang et al., 2014). To verify the reliability of the numerical methods adopted in this study, the inlet of computational domain was set as total mass flow rate and corresponding void fraction of the medium; the diameter of gas bubble in two-phase flow was given $0.4 \mathrm{~mm}$ according to the experimental value; while at the outlet, the static pressure was specified. The model used for the tip clearance of the rotor is General Connection interface model, which is a powerful way to connect regions together. In terms of the numerical solution, a frozen-stator method was given for the rotor-stator interfaces and the second order upwind scheme was used to solve the terms of advection and turbulence numerics. The total iterative step was set as 1000 and the RMS residual was set as $1 \times 10^{-4}$. Based on ANSYS_CFX16.0 code, the steady simulations for a gas-liquid two-phase pump with a full flow passage were carried out at conditions of pure water and four $I G V F s$, and compared with the experimental data, as shown in Figures 8 and 9.

As shown in Figure 8, the numerical efficiency and head at pure water conditions are in good agreement with the experimental results, especially the relative errors of efficiency and head which are only $0.94 \%$ and $2.97 \%$ at the nominal flow rate, respectively. While, as can be seen in Figure 9, the numerical heads at these four IGVF conditions have small errors compared to the experimental results, that is, errors of $3.00 \%, 2.07 \%, 3.30 \%$, and $1.57 \%$ for $I G V F s$ of $3 \%, 9 \%, 15 \%$, and $21 \%$, respectively. Therefore, it can be concluded that the numerical methods applied in the present study are reliable.

\subsubsection{Unsteady simulation}

To get the convergence results more readily, the steady results were taken as the initial values of the unsteady simulations. The method for rotor-stator interfaces was modified to "transient rotor-stator method", and it truly

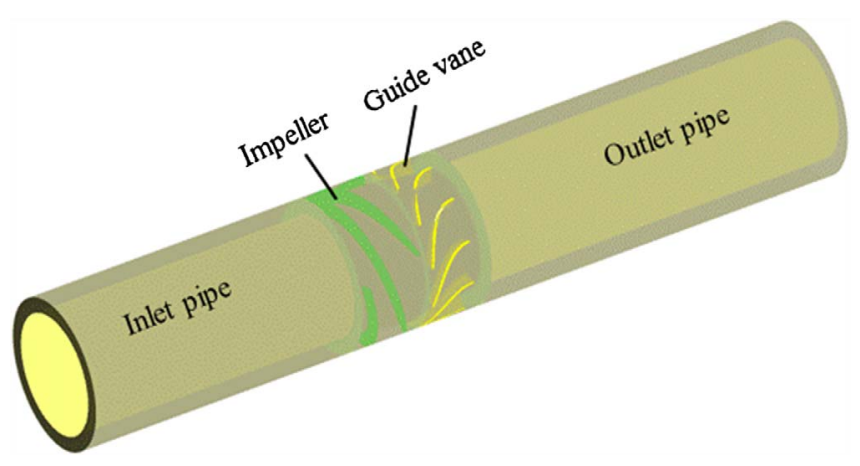

Fig. 5. Computational pump model.

reflects the transient effect and gives highly accurate predictions for the flow between rotor and stator.

To explore the pressure fluctuation in the impeller and guide vane passage, eight monitoring points denoted as $\mathrm{R} 1 \mathrm{C}-\mathrm{R} 4 \mathrm{C}, \mathrm{S} 1 \mathrm{C}-\mathrm{S} 4 \mathrm{C}$ were evenly arranged along the flow direction and located at mid-height in the radial direction, as shown in Figure 10. Meanwhile, at the unsteady simulation stage, the timescale plays a very important role in determining the numerical results and the computational speed. Therefore, at pure water design conditions, two time steps were analyzed - first $\Delta t_{1}=1.69 \times 10^{-4} \mathrm{~s}$ and then $\Delta t_{2}=1.13 \times 10^{-4} \mathrm{~s}$, corresponding to the time for the impeller rotation of $3^{\circ}$ and $2^{\circ}$ respectively, as presented in Table 3 and Figure 11. There is little difference in both the external characteristics of the pump (efficiency and head) and the pressure variation of monitoring points R4C, S1C, S4C at these two time steps. However, the efficiency and head of the pump in unsteady simulation are a bit larger than the steady results shown in Figure 7. This is because the unsteady simulation results given in Table 3 are extracted at a certain time, rather than the average values in the stable period. Compared to the single-phase flow, the flow with two phases is usually more disordered due to the phase interaction as well as the separation and the mixing. Therefore, $\Delta t_{2}=1.13 \times 10^{-4} \mathrm{~s}$ was chosen for the unsteady simulation at different $I G V F$ s. The total time was set as $0.203 \mathrm{~s}$, namely, the time that the impeller rotates 10 cycles.

\section{Results and discussion}

\subsection{Pressure fluctuation at condition of IGVF $=15 \%$}

Under conditions of $I G V F=15 \%$ and $Q=Q_{\mathrm{d}}$, the time domain characteristics of all points during one cycle are 


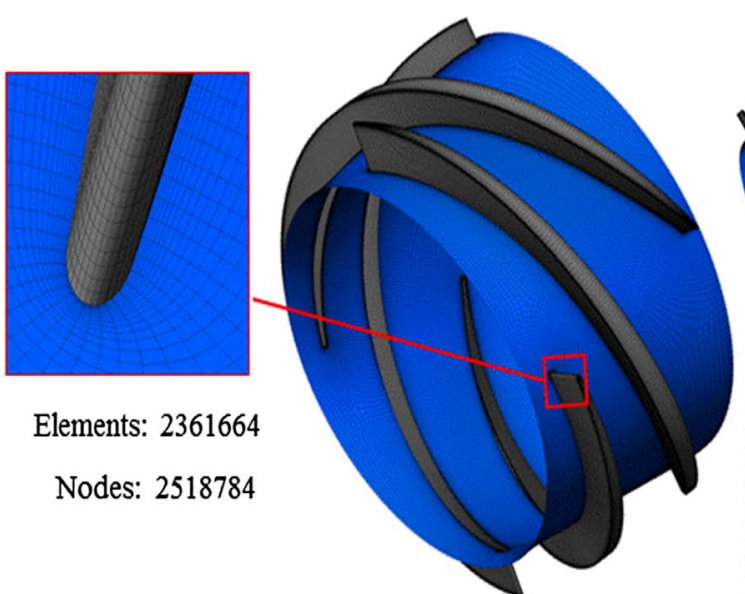

(a) impeller

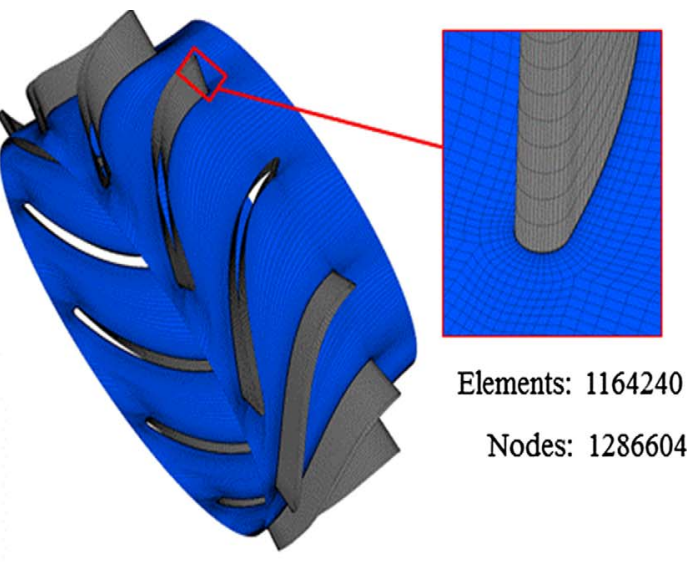

(b) guide vane

Fig. 6. Meshes for the (a) impeller and (b) guide vane.

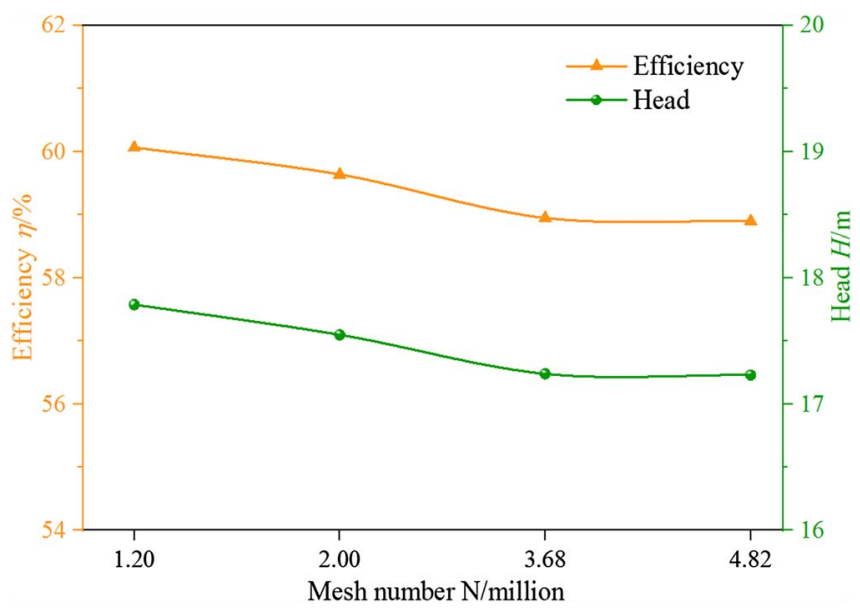

Fig. 7. Values of efficiency and head at different mesh numbers.

shown in Figure 12. The pressure fluctuations of points $\mathrm{R} 1 \mathrm{C}-\mathrm{R} 4 \mathrm{C}$ in the impeller are smaller than that of points $\mathrm{S} 1 \mathrm{C}-\mathrm{S} 4 \mathrm{C}$ in the guide vane. Also, an obvious cyclical fluctuation occurs for the points $\mathrm{R} 2 \mathrm{C}-\mathrm{R} 3 \mathrm{C}$ and points S1C-S4C, that is, there are eleven and four peaks and valleys respectively during one cycle which corresponds to the number of blades of guide vane and impeller, respectively.

Using the Fast Fourier Transform (FFT) method, the frequency domain characteristics of points $\mathrm{R} 1 \mathrm{C}-\mathrm{R} 4 \mathrm{C}$, $\mathrm{S} 1 \mathrm{C}-\mathrm{S} 4 \mathrm{C}$ are obtained, as shown in Figure 13. Along the streamwise direction, the fluctuation in the impeller increases firstly and then decreases, while there is a gradual decrease for the fluctuation in the guide vane and the maximum fluctuation amplitude for all points appears near the guide vane inlet (point S1C). The fluctuation near the rotor-stator interaction region, that is, from the impeller outlet to guide vane inlet regions is relatively large and the rotor-stator interaction region is usually taken as the main reason for the pressure fluctuation in single phase pumps. However, as can be seen in Figure 13, the fluctuation of points $\mathrm{R} 4 \mathrm{C}$ located near the impeller outlet is relatively small, which should go hand in hand with the gas-liquid two-phase flow therein. As there is a difference in density between the gas and liquid phases, the gas phase will gather at the outlet of the impeller (Fig. 14a) and form a gas vortex in the corresponding regions (Fig. 14b), which have been described in detail previously (Zhang et al., 2018b). Therefore, it can be drawn that the complex gasliquid two-phase flow will weaken the pressure fluctuation in the vicinity of the impeller outlet that has been attributed to the rotor-stator interaction.

It can be also seen from Figure 13 that, aside from the dominant frequency, there are more chaotic frequencies for the points in the impeller. This is associated with the complex gas-liquid two-phase flow caused by the rotation of the impeller. Additionally, the fluctuation in impeller was generally less than that in the guide vane, which shows that the rotor-stator interaction has a greater effect on the fluctuation in guide vane at $I G V F=15 \%$.

\subsection{Influence of Inlet Gas Void Fractions (IGVFs)}

The information about the dominant frequency of monitoring points at different $I G V F$ conditions are listed in Table 4, where, fn is the rotating frequency of impeller with a computed value of about $49 \mathrm{~Hz}$ based on the rotational speed of the pump. Overall, the dominant frequencies of all points are $11 \mathrm{fn}$ and $4 \mathrm{fn}$ respectively which corresponds to the blade numbers of guide vane and impeller. Therefore, it can be illustrated that the rotor-stator interaction is still responsible of the pressure fluctuation in the multiphase rotodynamic pump handling gas-liquid two-phase flow. The rotor-stator interaction is the interactions between the cyclical rotating impeller and the stationary guide vane. The forces acting on the impeller blades will change periodically when each blade rotates through one guide vane spacing.

From Table 4, the dominant frequency for the points in the impeller includes $0.5 \mathrm{fn}$ except $11 \mathrm{fn}$, which is believed to relate to the flow separation and the vortex shedding 
Table 2. Detailed mesh information for the impeller and guide vane.

\begin{tabular}{lcccccc}
\hline Item & Elements & Nodes & Orthogonality $\left(0^{\circ}-90^{\circ}\right)$ & Expansion factor $(1-20)$ & Aspect ratio $(1-100)$ & $y+$ \\
\hline Impeller & 2361664 & 2518784 & 82 & 1.2 & 13.9 & 66 \\
Guide vane & 1164240 & 1286604 & 74 & 4.9 & 18.2 & 10 \\
\hline
\end{tabular}

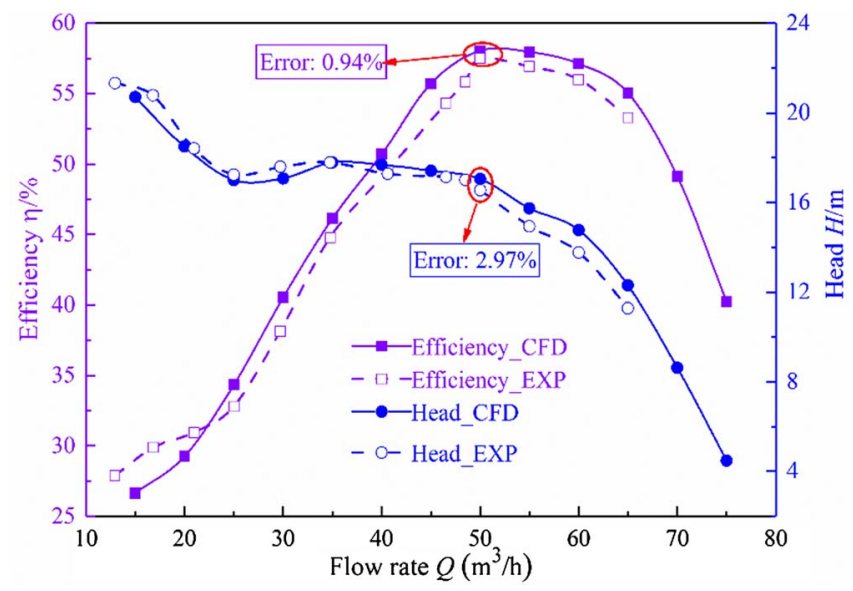

Fig. 8. Performance curves of efficiency and head for simulation and experiment at $I G V F=0 \%$.

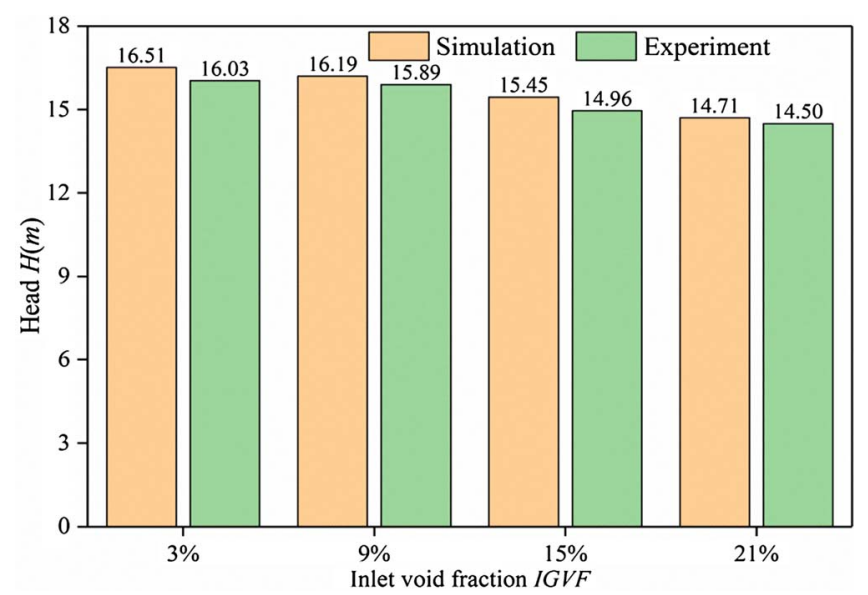

Fig. 9. Values of head for simulation and experiment at different $I G V F \mathrm{~s}\left(Q=Q_{\mathrm{d}}\right)$.

frequency in the corresponding area (Ni et al., 2018; Xu et al., 2017; Zhang et al., 2017b). The point R1C is located at the impeller inlet and is subjected to a weaker rotorstator interaction, thus the fluctuation caused by vortex separation will play a leading role at some conditions. However, where $I G V F=21 \%$, besides point $\mathrm{R} 1 \mathrm{C}$, the dominant frequency of point $\mathrm{R} 4 \mathrm{C}$ is also $0.5 \mathrm{fn}$ which is related to the accumulated gas in the impeller outlet and that increases significantly at $I G V F=21 \%$. This then results in a turbulent gas-liquid flow, which can be

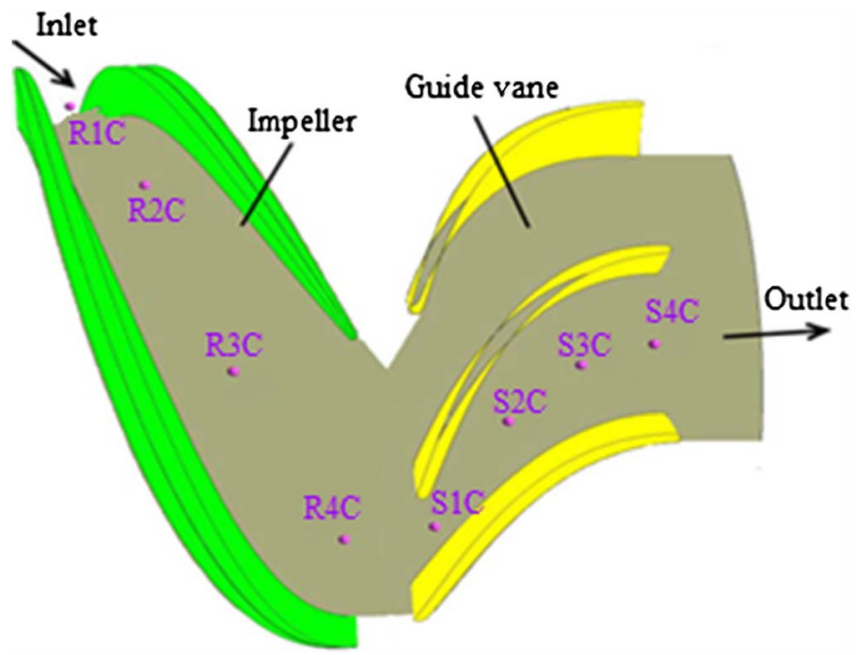

Fig. 10. Locations of monitoring points in the impeller and guide vane passages.

Table 3. Values of efficiency and head of the pump at two time steps $\left(Q=Q_{\mathrm{d}}\right)$.

\begin{tabular}{lcc}
\hline$\Delta t$ & Efficiency $\eta(\%)$ & Head $H(\mathrm{~m})$ \\
\hline$\Delta t_{1}$ & 60.28 & 17.72 \\
$\Delta t_{2}$ & 60.29 & 17.73 \\
\hline
\end{tabular}

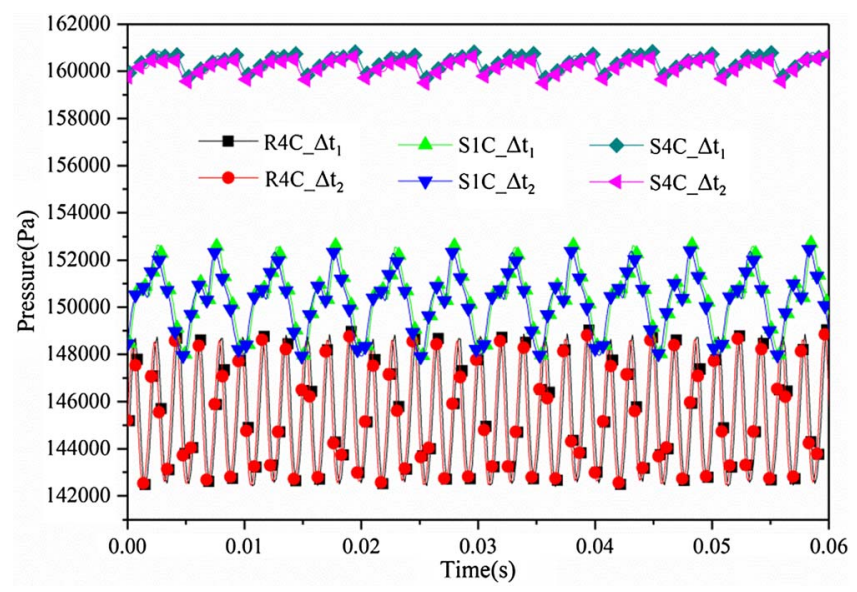

Fig. 11. Time domain characteristics of monitoring points $\mathrm{R} 4 \mathrm{C}, \mathrm{S} 1 \mathrm{C}, \mathrm{S} 4 \mathrm{C}$ at two time steps.

verified by the distributions of water superficial velocity and gas void fraction, as shown in Figures 15 and 16c, respectively. 


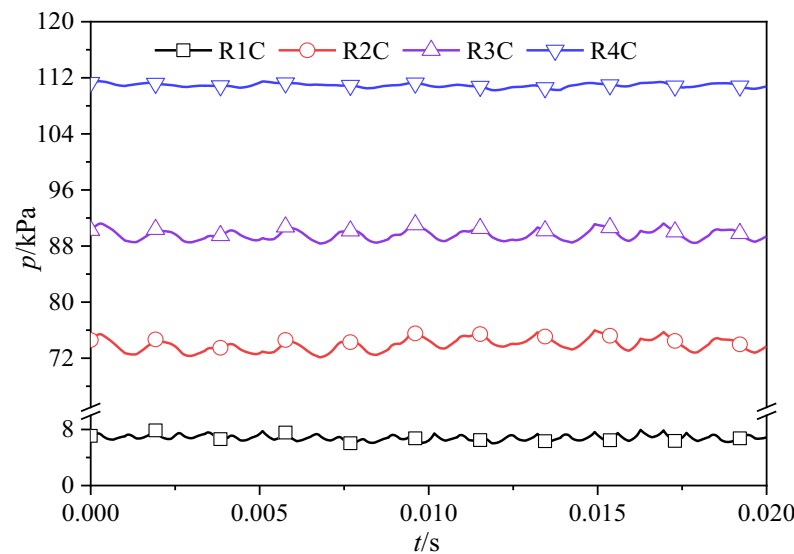

(a) Points $\mathrm{R} 1 \mathrm{C} \sim \mathrm{R} 4 \mathrm{C}$

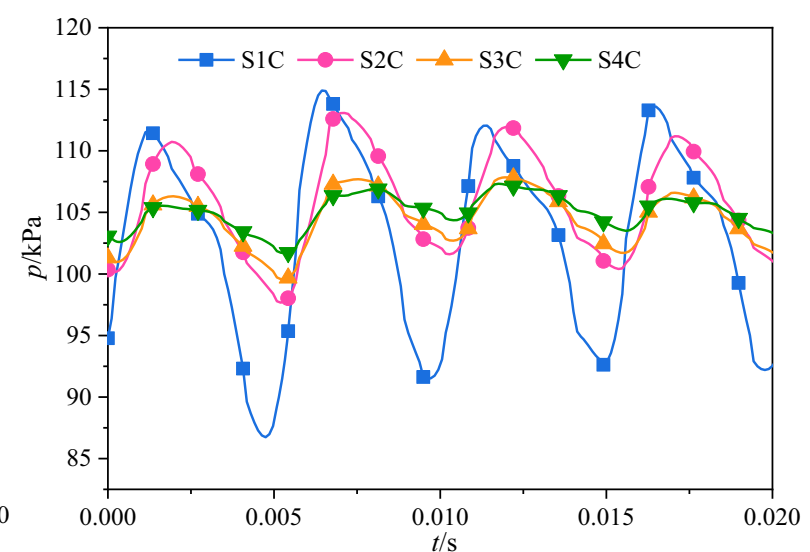

(b) Points S1C $\sim \mathrm{S} 4 \mathrm{C}$

Fig. 12. Time domain characteristics of points (a) R1C-R $4 \mathrm{C}$ and (b) $\mathrm{S} 1 \mathrm{C}-\mathrm{S} 4 \mathrm{C}$ at $I G V F=15 \%$ and $Q=Q_{\mathrm{d}}$.

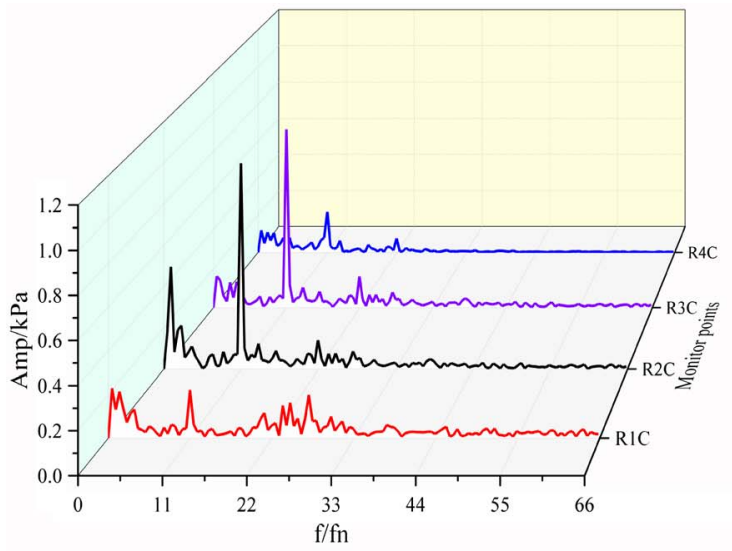

(a) points R1C-R4C

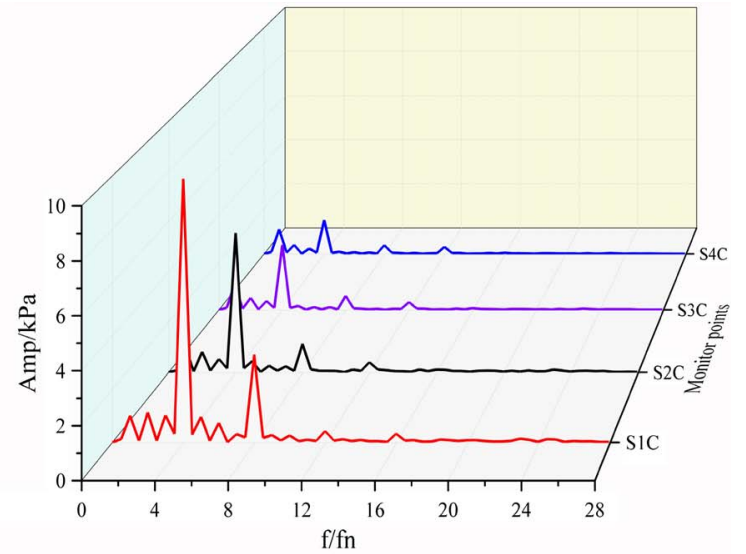

(b) points S1C-S4C

Fig. 13. Frequency domain characteristics of points (a) R1C-R4C and (b) S1C-S4C $\left(I G V F=15 \%, Q=Q_{\mathrm{d}}\right)$.

Table 5 lists the fluctuation coefficients of points where the fluctuation coefficient $C_{p}$ is defined as follows:

$$
\begin{gathered}
C_{p}=\frac{\sigma}{\rho_{l} \mathrm{~g} H_{\mathrm{d}}} \times 100 \%, \\
\sigma=\sqrt{\frac{1}{N} \sum_{i=1}^{N}\left[p_{i}(t)-\bar{p}_{i}\right]^{2},}
\end{gathered}
$$

where $H_{\mathrm{d}}$ is the designed pump head; $\rho_{l}$ is the density of water; and $p_{i}(t)$ and $\bar{p}_{i}$ represent the values of the instantaneous and average pressure, respectively.

From Table 5, the variation trend of fluctuation is similar at different $I G V F$ s along the streamwise direction, that is, (1) the fluctuation in the impeller increases firstly and then decreases, while it decreases gradually in the guide vane; (2) the fluctuation in the guide vane is greater than that in the impeller and the maximum fluctuation amplitude for all points appears near the inlet of the guide vane (point S1C); (3) on the whole, the fluctuation of point R4C in the vicinity of impeller outlet is relatively small among all eight points. The characteristics of the frequency domains for points $\mathrm{R} 4 \mathrm{C}$ and $\mathrm{S} 1 \mathrm{C}$ are shown in Figure 17. It can be seen that with the increase of $I G V F$, the influence of frequencies (except the dominant frequency) increases and more chaotic frequencies appear. This is closely related to the increase of the accumulated gas as the $I G V F$ increases, shown in Figure 17. The accumulated gas will block the passage and the flow will be more disordered.

Further, as the $I G V F$ increases, the fluctuation of the corresponding points shown in Table 5 overall first increases before decreasing, which illustrates that as the $I G V F$ increases within limits, a more disordered flow and enhanced gas-liquid phase interaction will occur, and resulting in greater pressure fluctuation. However, when 


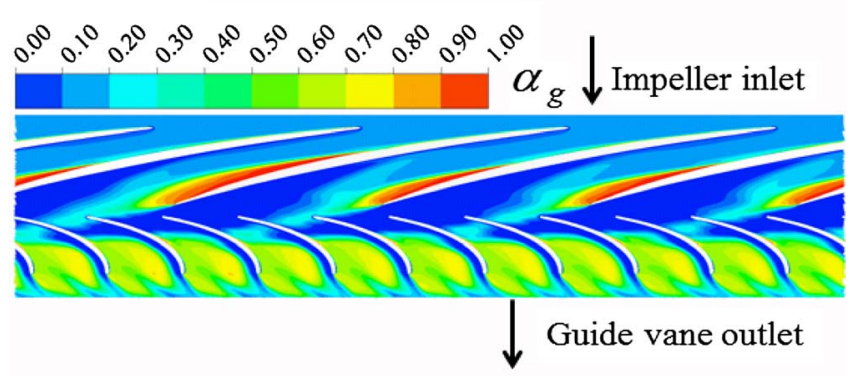

(a) distribution of gas void fraction

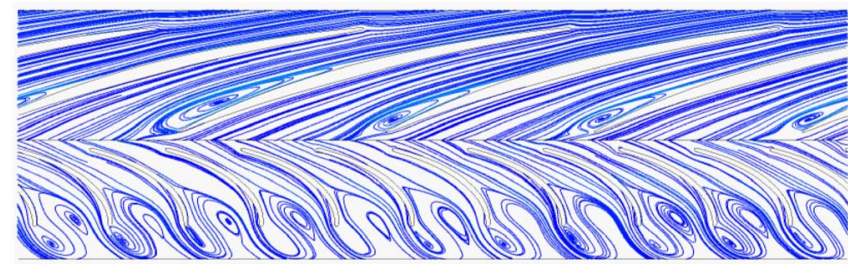

(b) distribution of gas streamlines

Fig. 14. Field characteristics for the blade to blade of the impeller and guide vane $\left(I G V F=15 \%, Q=Q_{\mathrm{d}}\right.$, span $\left.=0.5\right)$. (a) Distribution of gas void fraction, (b) distribution of gas streamlines.

Table 4. Information about the dominant frequency of points at different $I G V F_{\mathrm{s}}\left(Q=Q_{\mathrm{d}}\right)$.

\begin{tabular}{llllllllll}
\hline & \multicolumn{9}{c}{ Point } \\
\cline { 2 - 5 } \cline { 6 - 8 } IGVF & R1C & R2C & R3C & R4C & & S1C & S2C & S3C & S4C \\
\hline $3 \%$ & $0.5 \mathrm{fn}$ & $11 \mathrm{fn}$ & $11 \mathrm{fn}$ & $11 \mathrm{fn}$ & & $4 \mathrm{fn}$ & $4 \mathrm{fn}$ & $4 \mathrm{fn}$ & $4 \mathrm{fn}$ \\
$9 \%$ & $11 \mathrm{fn}$ & $11 \mathrm{fn}$ & $11 \mathrm{fn}$ & $11 \mathrm{fn}$ & $4 \mathrm{fn}$ & $4 \mathrm{fn}$ & $4 \mathrm{fn}$ & $4 \mathrm{fn}$ \\
$15 \%$ & $0.5 \mathrm{fn}$ & $11 \mathrm{fn}$ & $11 \mathrm{fn}$ & $11 \mathrm{fn}$ & $4 \mathrm{fn}$ & $4 \mathrm{fn}$ & $4 \mathrm{fn}$ & $4 \mathrm{fn}$ \\
$21 \%$ & $0.5 \mathrm{fn}$ & $11 \mathrm{fn}$ & $11 \mathrm{fn}$ & $0.5 \mathrm{fn}$ & $4 \mathrm{fn}$ & $4 \mathrm{fn}$ & $4 \mathrm{fn}$ & $4 \mathrm{fn}$ \\
\hline
\end{tabular}

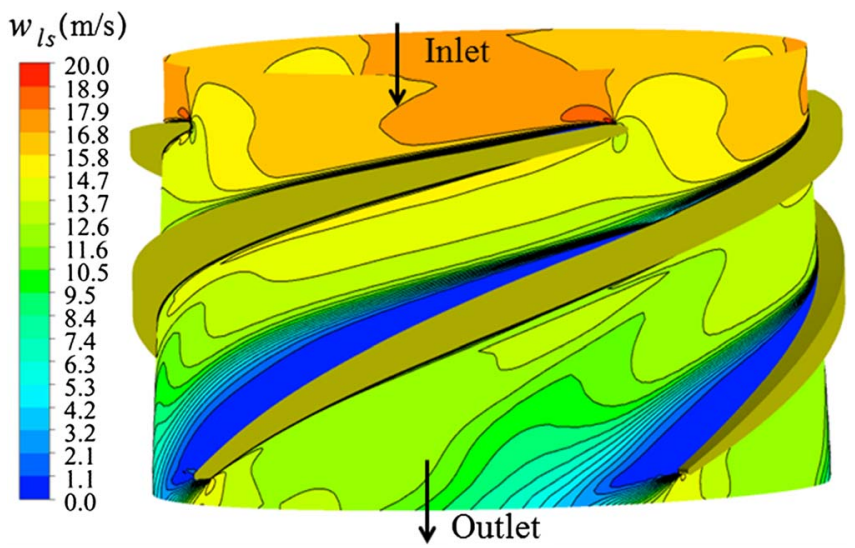

Fig. 15. Distribution of water superficial velocity in the impeller at $I G V F=21 \%(\operatorname{span}=0.5)$.

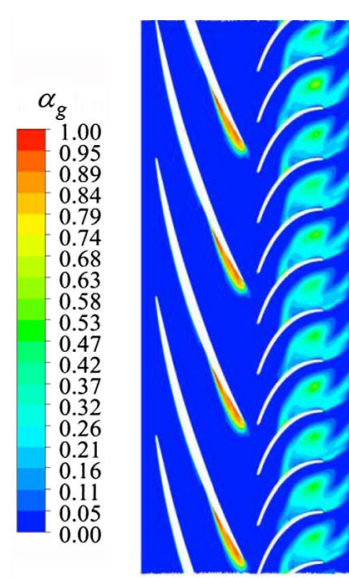

(a) $I G V F=3 \%$

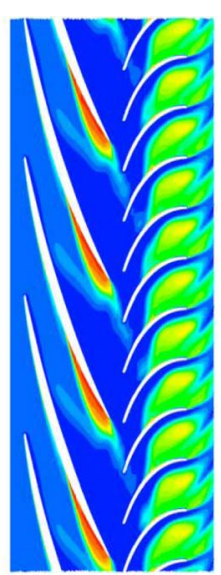

(b) $I G V F=9 \%$

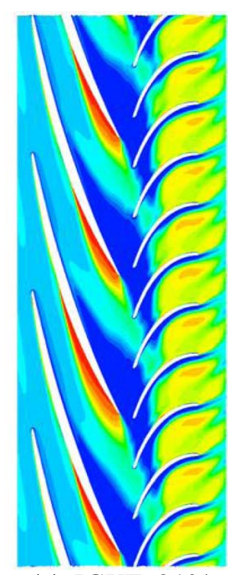

(c) $I G V F=21 \%$
Fig. 16. Distribution of $G V F$ in impeller and guide vane at different $I G V F \mathrm{~s}$ (span $=0.5, Q=Q_{\mathrm{d}}$ ). (a) $I G V F=3 \%$, (b) $I G V F=9 \%$, (c) $I G V F=21 \%$.

Table 5. Pressure fluctuation coefficients of points at different $I G V F \mathrm{~s}\left(\%, Q=Q_{\mathrm{d}}\right)$.

\begin{tabular}{|c|c|c|c|c|c|c|c|c|}
\hline \multirow[b]{3}{*}{$I G V F$} & \multicolumn{8}{|c|}{ Point } \\
\hline & \multicolumn{4}{|c|}{ Impeller } & \multicolumn{4}{|c|}{ Guide vane } \\
\hline & $\mathrm{R} 1 \mathrm{C}$ & $\mathrm{R} 2 \mathrm{C}$ & $\mathrm{R} 3 \mathrm{C}$ & $\mathrm{R} 4 \mathrm{C}$ & $\mathrm{S} 1 \mathrm{C}$ & $\mathrm{S} 2 \mathrm{C}$ & $\mathrm{S} 3 \mathrm{C}$ & $\mathrm{S} 4 \mathrm{C}$ \\
\hline $3 \%$ & 0.31 & 0.50 & 0.39 & 0.41 & 2.99 & 2.02 & 1.06 & 0.57 \\
\hline $9 \%$ & 0.32 & 0.64 & 0.59 & 0.34 & 4.46 & 2.71 & 1.36 & 0.82 \\
\hline $15 \%$ & 0.35 & 0.61 & 0.50 & 0.19 & 5.17 & 2.84 & 1.50 & 0.95 \\
\hline $21 \%$ & 0.25 & 0.45 & 0.40 & 0.18 & 5.18 & 2.70 & 1.45 & 0.87 \\
\hline
\end{tabular}

the $I G V F$ is outside of the limit, the degree of gas accumulation will be clearly enhanced and thus the gas mass forms, as shown in Figure 16c, thus decreasing the collision probabilities between the gas-liquid molecules. According to the research of Konno et al. (2001), part of the energy released from the molecular collision may be absorbed by the gas mass, thus leading to the decrease of pressure fluctuation. 


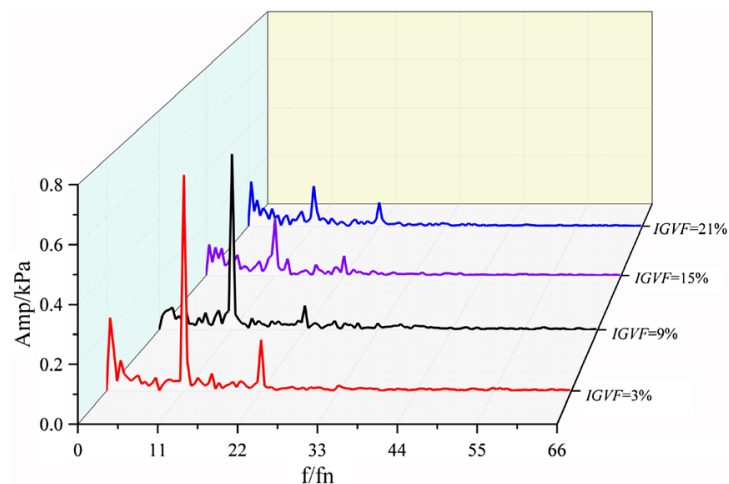

(a) point $\mathrm{R} 4 \mathrm{C}$

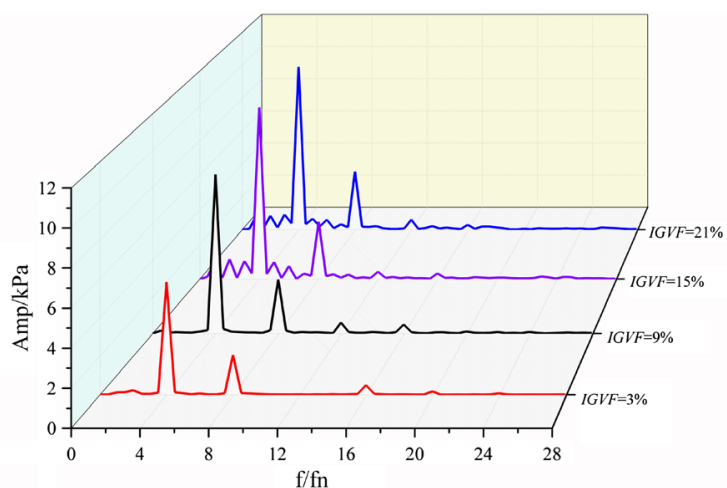

(b) point S1C

Fig. 17. Frequency domain characteristics of points (a) R4C and (b) S1C at different $I G V F \mathrm{~s}\left(Q=Q_{\mathrm{d}}\right)$.

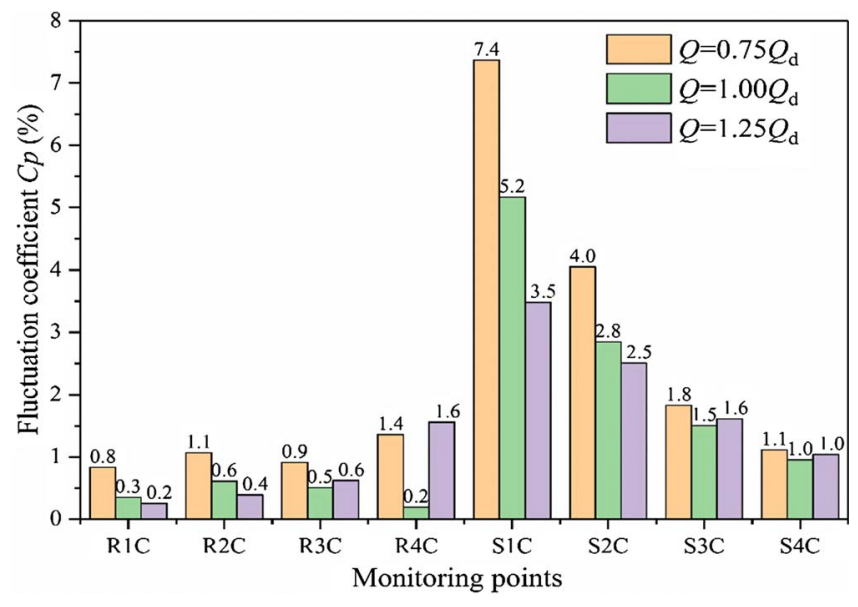

Fig. 18. Fluctuation coefficient of points at different flow rates $(I G V F=15 \%)$.

\subsection{Influence of flow rates}

Figure 18 shows the pressure fluctuation of points at different flow rates $\left(0.75 Q_{\mathrm{d}}, Q_{\mathrm{d}}, 1.25 Q_{\mathrm{d}}\right)$ and an $I G V F$ of $15 \%$. Overall, the fluctuation in the impeller along the streamwise direction first increases and then decreases at $Q=Q_{\mathrm{d}}$, and increases gradually under $0.75 Q_{\mathrm{d}}$ and $1.25 Q_{\mathrm{d}}$ conditions, while the fluctuation in the guide vane decreases gradually at these three flow rates. Furthermore, the influence of flow rate on the fluctuation is not consistent in the vicinity of the interaction region (points R3C, R4C, S1C, $\mathrm{S} 2 \mathrm{C}$ ). For points S1C, S2C, the fluctuations generally decrease with increasing flow rate due to the entrainment capacity of the liquid which is enhanced as the flow rate increases, thus decreasing the degree of the accumulation of gas and the flow separation between the gas-liquid phases. But for points R3C, R4C, due to the inconsistency between the inflow angle and the blade angle at off-design condition, the fluctuations at conditions of $Q=0.75 Q_{\mathrm{d}}$

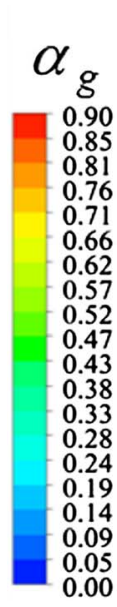

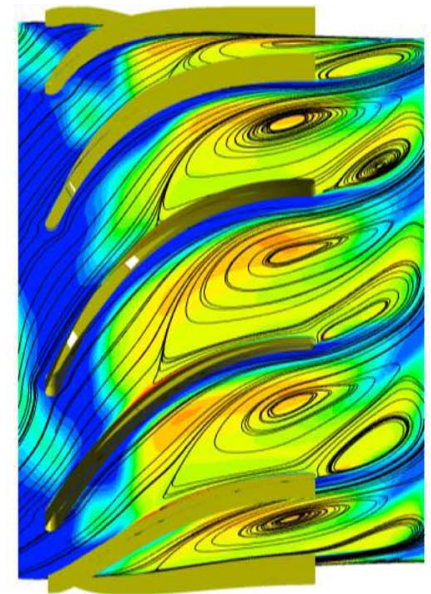

(a) $Q=0.75 Q_{\mathrm{d}}$

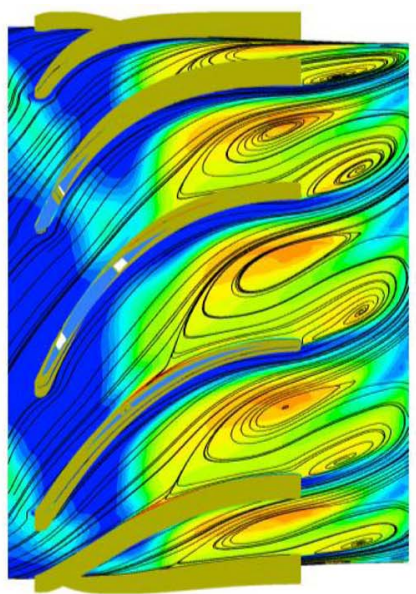

(b) $Q=Q_{\mathrm{d}}$

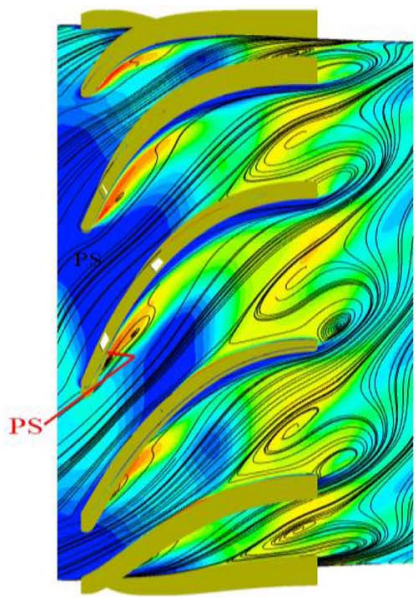

(c) $Q=1.25 Q_{\mathrm{d}}$

Fig. 19. Distributions of $G V F$ and gas streamlines in guide vane at different flow rates $(I G V F=15 \%$, span $=0.5)$. (a) $Q=0.75 Q_{\mathrm{d}}$, (b) $Q=Q_{\mathrm{d}}$, (c) $Q=1.25 Q_{\mathrm{d}}$. 


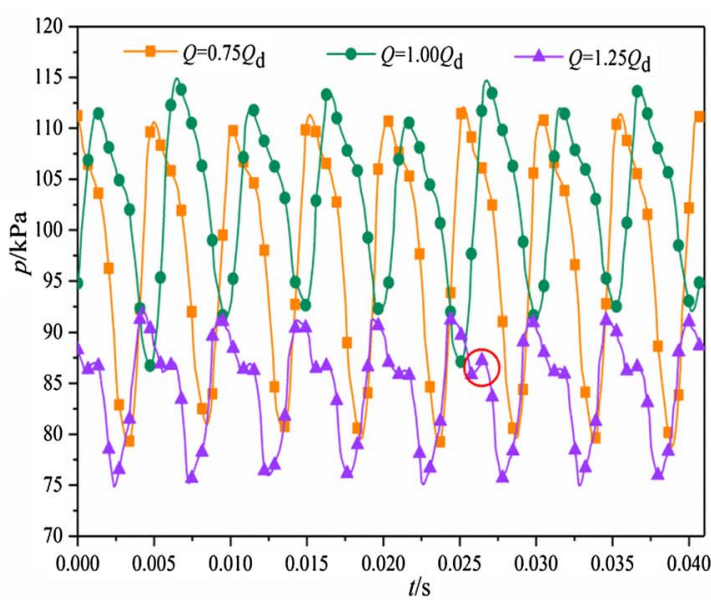

(a) time domain

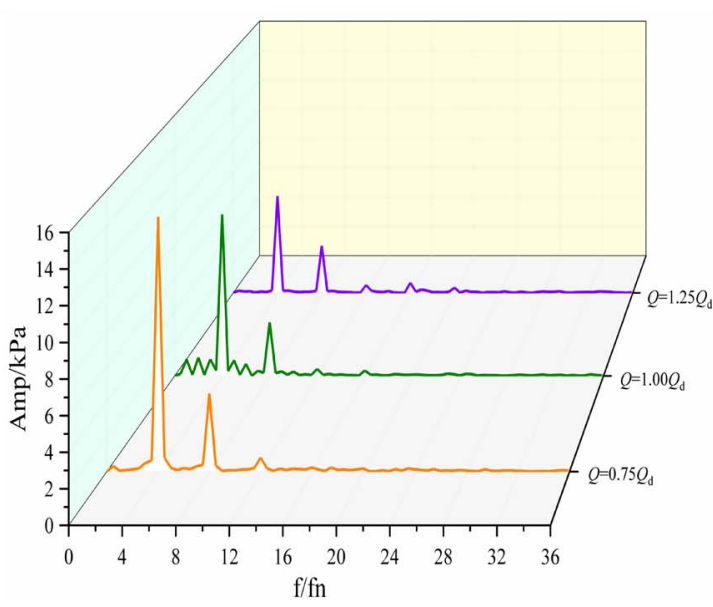

(b) frequency domain

Fig. 20. Characteristics of (a) time and (b) frequency domains for point S1C at different flow rates $(I G V F=15 \%)$.

and $Q=1.25 Q_{\mathrm{d}}$ are greater than the condition of $Q=Q_{\mathrm{d}}$. The same phenomenon can be observed at the trailing edge of the guide vane (points $\mathrm{S} 3 \mathrm{C}, \mathrm{S} 4 \mathrm{C}$ ), this can also be attributed to the off-design effect at the guide vane inlet. However, a strong flow separation occurs at the trailing edge of the guide vane (shown in Fig. 19), thus the impact of the effect is weak and the difference of fluctuation at the three flow rates is relatively small.

The analysis shown in Figure 18 also shows that the fluctuation in the guide vane is greater than for the impeller at these three flow rates and the greatest degree of fluctuation occurs at point S1C. Therefore, the characteristics of the time and frequency domains for point S1C were analyzed and are shown in Figure 20. Four peaks and valleys occur in one cycle and the dominant frequency and second dominant frequency are $4 \mathrm{fn}$ and $8 \mathrm{fn}$ respectively, which corresponds to the blade number of the impeller or its integer multiple.

Figure 20a shows that the phenomenon of the second peak and valley occurs at $Q=1.25 Q_{\mathrm{d}}$, marked with a red circle, which is attributed to the rotor-stator interaction. That is, the pressure wave generated by the rotation of the impeller propagates along the flow direction and then generates the refraction and the reflection after colliding with the pressure surface of the guide vane blades. Therefore, the gas-liquid two-phase flow near the pressure surface (PS) at the inlet of the guide vane will be more disordered, as shown in Figure 19c, and the accumulation of gas and the flow separation occur therein.

\section{Conclusion}

The purpose of this study was to investigate the main reason for the generation of pressure fluctuation, as well as their change rules, in a multiphase rotodynamic pump handling air-water two-phase flow, which will help in the optimization design for such pumps. Based on the Euler two-fluid model, the pressure fluctuations in the impeller and guide vane passages were analyzed at different $I G V F \mathrm{~s}$ and various flow rates. The main conclusions were:

Along streamwise flow direction, the fluctuation in the impeller first increases and then decreases at different $I G V F_{\mathrm{s}}$, while it decreases gradually for that in the guide vane. Also, the complex gas-liquid flow in the vicinity of the impeller outlet weakens the fluctuation therein. Additionally, as $I G V F$ increases, the fluctuation of the corresponding points overall first increases and then decreases.

When the $I G V F$ is $15 \%$, along the streamwise direction, the fluctuation in the impeller overall first increases and then decreases at $Q=Q_{\mathrm{d}}$, and increases gradually under $0.75 Q_{\mathrm{d}}$ and $1.25 Q_{\mathrm{d}}$ conditions, while the fluctuation in the guide vane decreases gradually at these three flow rates. Also, the phenomenon of the second peak and valley for point SIC located near the inlet of the guide vane is seen when $Q=1.25 Q_{\mathrm{d}}$.

Overall, the dominant frequency of points are respectively $11 \mathrm{fn}$ and $4 \mathrm{fn}$, corresponding to the number of blades of the guide vane and impeller. This illustrates that the rotor-stator interaction can still be considered the main reason for the generation of fluctuation in gas-liquid twophase pumps. Furthermore, the effect of rotor-stator interaction on the fluctuation in the guide vane is greater than for the impeller and the maximum fluctuation amplitude appears in the vicinity of the guide vane inlet (point S1C).

Acknowledgments. This study was supported by the National Natural Science Foundation of China (grant number 51579006), the Basic Research Foundation of Beijing Institute of Technology (grant number 20150342012).

\section{References}

Cao S.L., Peng G.Y., Yu Z.Y. (2005) Hydrodynamic design of rotodynamic pump impeller for multiphase pumping by combined approach of inverse design and CFD analysis, $J$. Fluids Eng. 127, 330-338. 
Caridad J., Kenyery F. (2004) CFD analysis of electric submersible pumps (ESP) handling two-phase mixtures, $J$. Energy Resour. 126, 99-104.

Caridad J., Asuaje M., Kenyery F., Tremante A., Aguillon O. (2008) Characterization of a centrifugal pump impeller under two-phase flow conditions, J. Pet. Sci. Eng. 63, 18-22.

Chen E., et al. (2016) Numerical investigation on vibration and noise induced by unsteady flow in an axial-flow pump, J. Mech. Sci. Technol. 30, 5397-5404.

Ding H., Visser F.C., Jiang Y., Furmanczyk M. (2011) Demonstration and validation of a $3 \mathrm{D}$ CFD simulation tool predicting pump performance and cavitation for industrial applications, J. Fluids Eng. 133, 1-13.

Feng J.J., Benra F.K., Dohmen H.J. (2010) Application of different turbulence models in unsteady flow simulations of a radial diffuser pump, Forsch. Ingenieurwes. 74, 123-133.

Feng J.J., Luo X., Guo P., Wu G. (2016) Influence of tip clearance on pressure fluctuations in an axial flow pump, $J$. Mech. Sci. Technol. 30, 1603-1610.

Franke M. (2009) Aspects of numerical simulation of turbomachinery flows, Eingeladener Vortrag am Institut für Technische und Angewandte Mechanik der TU Warschau, SAS IP Inc., Polen.

González J., Parrondo J., Santolaria C., Blanco E. (2006) Steady and unsteady radial forces for a centrifugal pump with impeller to tongue gap variation, J. Fluids Eng. 128, 454-462.

Hayashi I., Kaneko S. (2014) Pressure pulsations in piping system excited by a centrifugal turbomachinery taking the damping characteristics into consideration, J. Fluids Struct. 45, 216-234.

Kim J.H., et al. (2015) Improvement of hydrodynamic performance of a multiphase pump using design of experiment techniques, J. Fluids Eng. 137, 1-15.

Kocaaslan O., Ozgoren M., Babayigit O., Aksoy M.H. (2017) Numerical investigation of the effect of number of blades on centrifugal pump performance, American Institute of Physics Conference Series 1863, 1-4.

Konno A., Kato H., Yamaguchi H., Maeda M. (2001) On the collapsing behavior of cavitation bubble clusters, JSME Int. J. 45, 631-637.

Lane G.L., Schwarz M.P., Evans G.M. (2005) Numerical modelling of gas-liquid flow in stirred tanks, Chem. Eng. Sci. 60, 2203-2214.

Liu X.B., Hu Q.Y., Shi G.T., Zeng Y.Z., Wang H.Y. (2017a) Research on transient dynamic characteristics of three-stage axial-flow multi-phase pumps influenced by gas volume fractions, Adv. Mech. Eng. 9, 1-10.

Liu Y., Tan L., Liu M., Hao Y., Xu Y. (2017b) Influence of prewhirl angle and axial distance on energy performance and pressure fluctuation for a centrifugal pump with inlet guide vanes, Energies 10, 1-14.

Ma Y., Ni Y., Zhang H., Zhou S., Deng H. (2018) Influence of valve's lag characteristic on pressure pulsation and performance of reciprocating multiphase pump, J. Pet. Sci. Eng. 164, 584-594.

Majidi K. (2005) Numerical study of unsteady flow in a centrifugal pump, J. Turbomach. 127, 805-814.

Menter F.R. (1994) 2-equation eddy-viscosity turbulence models for engineering applications, AIAA J. 32, 1598-1605.

Minemura K., Murakami M. (1980) A theoretical-study on air bubble motion in a centrifugal pump impeller, J. Fluids Eng. 102, 446-455.
Minemura K., Uchiyama T. (1993) 3-dimensional calculation of air-water 2-phase flow in centrifugal pump impeller based on a bubbly flow model, J. Fluids Eng. 115, 766-771.

Miorini R.L., Wu H., Katz J. (2012) The internal structure of the tip leakage vortex within the rotor of an axial waterjet pump, J. Turbomach. 134, 403-419.

Miyabe M., Maeda H., Umeki I., Jittani Y. (2006) Unstable head-flow characteristic generation mechanism of a low specific speed mixed flow pump, J. Therm. Sci. 15, 115-120.

Ni Y., Zhu R., Zhang X., Pan Z. (2018) Numerical investigation on radial impeller induced vortex rope in draft tube under partial load conditions, J. Mech. Sci. Technol. 32, 157165.

Nishida M., et al. (2016) Long-term durability test of axial-flow ventricular assist device under pulsatile flow, J. Artif. Organs 20, 1-8.

Pineda H., et al. (2016) Phase distribution analysis in an Electrical Submersible Pump (ESP) inlet handling water-air two-phase flow using Computational Fluid Dynamics (CFD), J. Pet. Sci. Eng. 139, 49-61.

Qu W.S., Tan L., Cao S.L., Wang Y.C., Xu Y. (2016) Numerical investigation of clocking effect on a centrifugal pump with inlet guide vanes, Eng. Comput. 33, 465-481.

SAS IP, Inc (2013) ANSYS TurboGrid Users Guide, Release 15.0, Pittsburgh, PA, USA.

Shuai Z.J., Li W.Y., Zhang X.Y., Jiang C.X., Li F.C. (2014) Numerical study on the characteristics of pressure fluctuations in an axial-Flow water pump, Adv. Mech. Eng. 2, 1-7.

Stel H., Sirino T., Ponce F.J., Chiva S., Morales R.E.M. (2015) Numerical investigation of the flow in a multistage electric submersible pump, J. Pet. Sci. Eng. 136, 41-54.

Suh J.W., et al. (2017) Multi-objective optimization of the hydrodynamic performance of the second stage of a multiphase pump, Energies 10, 1-21.

Suh J.W., et al. (2018) Development of numerical EulerianEulerian models for simulating multiphase pumps, J. Pet. Sci. Eng. 162, 588-601.

Tan L., Zhu B.S., Cao S.L., Wang Y.C., Wang B.B. (2014) Influence of prewhirl regulation by inlet guide vanes on cavitation performance of a centrifugal pump, Energies 7, 1050-1065.

Tan L., Zhang J.S. (2018) Energy performance and pressure fluctuation of a multiphase pump with different gas volume fractions, Energies 11, 1-14.

Xie C., et al. (2018) Numerical calculation of axial-flow pump's pressure fluctuation and model test analysis, Adv. Mech. Eng. 10, 1-13.

Xu Y., Tan L., Liu Y., Cao S. (2017) Pressure fluctuation and flow pattern of a mixed-flow pump with different blade tip clearances under cavitation condition, Adv. Mech. Eng. 9, 1-12.

Yakhot V., Orszag S.A., Thangam S., Gatski T.B., Speziale C.G. (1992) Development of turbulence models for shear Flows by a double expansion technique, Phys. Fluids A-Fluid Dyn. 4, 1510-1520.

Yan D., Kovacevic A., Tang Q., Rane S., Zhang W.H. (2017) Numerical modelling of twin-screw pumps based on computational fluid dynamics, Proc. Inst. Mech. Eng., Part C: J. Mach. Eng. Sci. 231, 4617-4634.

Yu Z.Y., Zhu B.S., Cao S.L., Liu Y. (2014) Effect of virtual mass force on the mixed transport process in a multiphase rotodynamic pump, Adv. Mech. Eng. 2, 1-7.

Yu Z.Y., Zhu B.S., Cao S.L. (2015) Interphase force analysis for air-water bubbly flow in a multiphase rotodynamic pump, Eng. Comput. 32, 2166-2180. 
Zhang K.W. (2000) Theory of fluid machinery, Vol. 1, China Machine Press, Beijing, China.

Zhang Y., Zhang J., Zhu H., Cai S. (2014) 3D blade hydraulic design method of the rotodynamic multiphase pump impeller and performance research, Adv. Mech. Eng. 1, 1-10.

Zhang J., Cai S., Zhu H., Zhang Y. (2015) Experimental investigation of the flow at the entrance of a rotodynamic multiphase pump by visualization, J. Pet. Sci. Eng. 126, 254-261.

Zhang W., Yu Z., Zhu B. (2017a) Influence of tip clearance on pressure fluctuation in low specific speed mixed-flow pump passage, Energies 10, 1-16.

Zhang W., Zhu B., Yu Z., Yang C. (2017b) Numerical study of pressure fluctuation in the whole flow passage of a low specific speed mixed-flow pump, Adv. Mech. Eng. 9, 1-11.
Zhang W.W., Yu Z.Y., Zhu B.S. (2017c) Numerical study of pressure fluctuation in a gas-liquid two-phase mixed-flow pump, Energies 10, 1-14.

Zhang W.W., Yu Z.Y., Li Y.J. (2018a) Analysis of flow and phase interaction characteristics in a gas-liquid two-phase pump, Oil Gas Sci. Technol. - Rev. IFP Energies nouvelles 73, 69.

Zhang W.W., Yu Z.Y., Zahid M.N., Li Y.J. (2018b) Study of the gas distribution in a multiphase rotodynamic pump based on interphase force analysis, Energies 11, 1-16.

Zhang W.W., Yu Z.Y., Li Y.J. (2019) Application of a nonuniform bubble model in a multiphase rotodynamic pump, $J$. Pet. Sci. Eng. 173, 1316-1322.

Zhu J.J., Zhang H.Q. (2016) Mechanistic modeling and numerical simulation of in-situ gas void fraction inside ESP impeller, J. Nat. Gas Sci. Eng. 36, 144-154. 